\title{
Performance of EuroSCORE and EuroSCORE II in Institut Jantung Negara (IJN), Kuala Lumpur, Malaysia
}

\author{
M. Azizan Petra ${ }^{*}$, M. Ezani Taib, M. F. Ramli, N. Jaafar, I. F. Gaafar
}

Institut Jantung Negara, Kuala Lumpur, Malaysia

DOI: $10.36347 /$ sjams.2020.v08i06.001

| Received: 05.05.2020 | Accepted: 23.05.2020 | Published: 08.06.2020

*Corresponding author: M. Azizan Petra

Objective: Assessing the performance of European System for Cardiac Operative Evaluation (EuroSCORE) and EuroSCORE II. Method: 4145 patients who underwent cardiac surgery between $1^{\text {st }}$ January 2015 to $31^{\text {st }}$ December 2016 in Institut Jantung Negara (IJN) were included. The entire cohort and isolated coronary bypass graft (CABG) patients were analyzed by measuring the area under the receiver operating characteristic (ROC) curve for model discrimination and Hosmer-Lemeshow Chi-squared test for model calibration. Performance of both models was compared. Result: For the entire cohort, ROC curve for EuroSCORE was 0.679; EuroSCORE II was 0.615. For isolated CABG patients, ROC curve for EuroSCORE was 0.670; EuroSCORE II was 0.609. For the entire cohort, Hosmer-Lemeshow test showed no significant difference between expected and observed mortality according to EuroSCORE model (Chi-square $=5.284, \mathrm{P}=0.508$ ) and EuroSCORE II model (Chi-square $=15.828, \mathrm{P}=0.050)$. For the isolated CABG patients, Hosmer-Lemeshow test showed no significant difference between expected and observed mortality according to EuroSCORE model (Chi-square $=5.365, \mathrm{P}=0.498)$ and EuroSCORE II model $($ Chi-square $=$ 9.839, $\mathrm{P}=0.276$ ). For the entire cohort (Table 7), the observed and predicted mortality were $4.56 \%$ and $3.7 \%$ respectively for EuroSCORE; observed and predicted mortality were similar at $4.56 \%$ for EuroSCORE II. For isolated CABG patients (Table 8), the observed and predicted mortality were $3.62 \%$ and $3.36 \%$ respectively for EuroSCORE; the observed and predicted mortality were $3.62 \%$ and $3.97 \%$ respectively for EuroSCORE II. Conclusion: Despite poor discrimination under the ROC, the calibration of both models was good and acceptable to be used for risk prediction tools in our centre.

Keywords: EuroSCORE, receiver operating characteristic (ROC), predicted mortality.

Copyright @ 2020: This is an open-access article distributed under the terms of the Creative Commons Attribution license which permits unrestricted use, distribution, and reproduction in any medium for non-commercial use (NonCommercial, or CC-BY-NC) provided the original author and source are credited.

\section{INTRODUCTION}

Risk stratification and prediction models has become paramount tools in current cardiac surgical practice. These tools have been consistently used to help in clinical decision making, informed consent, quality assurance and healthcare management [1, 6]. These models are also useful to allow surgeons to compare mortality and outcomes with international data.

There are several risk predictions models that are used internationally; EuroSCORE, EuroSCORE II, Parsonnet, et cetera. These models have been tested and widely used internationally. It has been validated with good results in European and North American population $[1,5]$. These models are useful to predict patient outcome endpoints including long term mortality risk, intensive care unit stay, surgical complications and costs of cardiac surgery [1]. These models are based on Caucasian population and hence are most applicable when the demographics of preoperative patients are similar to the population which were used in the risk scoring models. Therefore, the risk scoring models may not be useful in some parts of the world. Because of this, risk scoring system can only be reliably used when its validity and performance has been tested in the centre of application.

This study is aimed at assessing the performance of European System for Cardiac Operative Evaluation (EuroSCORE) and EuroSCORE II in IJN.

\section{Study Design}

A retrospective single centre study was performed. Patients included are those who underwent cardiac surgery in Insititut Jantung Negara, Kuala Lumpur, Malaysia between $1^{\text {st }}$ January 2015 to 31 December 2016. Patients under 18 years old, closed heart surgery and transplant procedures are excluded from the study. Baseline characteristics of all included 
M. Azizan Petra et al., Sch J App Med Sci, June, 2020; 8(6): 1390-1396

patients are measured. The baseline characteristics used as risk factors for Euroscore and Euroscore II. This included the entire cohort and a subgroup of patients undergoing coronary artery bypass surgery (CABG) only were analysed.

Statistical analysis was performed by chisquare test. Data analysis was performed using SPSS 11.5 statistical software package. Observed and predicted mortality was compared in order to assess the performance of both models.

\section{Data Analysis}

The performances of the Euroscore models were analysed focusing on discrimination and calibration. The discrimination performance indicates the extent to which each model differentiates which patient will die or survive in the perioperative period. It was measured using receiver operating characteristic curve. Area under the curve were calculated with $95 \%$ confidence interval. An area of 0.5 reflects no discrimination and an area of 1.0 reflects perfect predictor. Areas of greater than 0.7 are generally thought to be useful [10].

Calibration refers to the agreement between observed outcomes and prediction. For example, 20 in hospital deaths should be observed in a 100 patients group with $20 \%$ predicted mortality. We used the Hosmer - Lemeshow Chi Square statistic to measure these values over deciles of risks. $\mathrm{P}$ value greater than 0.05 shows a well calibrated model [1].

\section{RESULTS}

Total of 6118 patients underwent open heart surgery during the study year period. 1973 patients were excluded leaving 4145 patients for analysis and out of this, 2820 patients underwent isolated CABG.

Table-1: Total patient breakdown before and after exclusion

\begin{tabular}{|l|l|l|l|l|}
\hline \multicolumn{2}{|l|}{} & $\mathbf{2 0 1 5}$ & $\mathbf{2 0 1 6}$ & Total \\
\hline Before exclusion & All & 3032 & 3086 & 6118 \\
\hline & Isolated CABG & 1383 & 1549 & 2932 \\
\hline After exclusion & All & 1984 & 2161 & 4145 \\
\hline & Isolated CABG & 1314 & 1506 & 2820 \\
\hline
\end{tabular}

The baseline variables and characteristics are measured for both groups for EuroScore and Euroscore II which are shown in Table-2. Most patient have normal $\mathrm{EF}$ in the overall and isolated $\mathrm{CABG}$ group; $50.1 \%$ and $45.5 \%$ respectively. Also, most patients underwent elective procedures in both groups; $70.2 \%$ and $69 \%$ respectively. It is important to note that there is a group of patients in the isolated $\mathrm{CABG}$, who were planned to have multiple procedures but ended up to have isolated $\mathrm{CABG}$.

Table-2: Baseline variables and characteristics are measured for both groups for EuroScore and Euroscore II.

\begin{tabular}{|c|c|c|c|c|}
\hline & \multicolumn{2}{|l|}{ Overall } & \multicolumn{2}{|l|}{ Isolated cabg } \\
\hline Risk factors & EuroSCORE & EuroSCORE II & EuroSCORE & EuroSCORE II \\
\hline age - mean \pm sd (years) & \multicolumn{2}{|c|}{$59.3 \pm 11.8$} & \multicolumn{2}{|l|}{$61.7 \pm 8.6$} \\
\hline median (iqr) & \multicolumn{2}{|c|}{$61.2(53.7,67.4)$} & \multicolumn{2}{|c|}{$62.2(56.1,67.8)$} \\
\hline gender (female) & \multicolumn{2}{|c|}{$1016(24.5)$} & \multicolumn{2}{|l|}{$455(16.1)$} \\
\hline creatinine & & & & \\
\hline $\mathrm{CC}<50$ & \multicolumn{2}{|l|}{$835(20.1)$} & \multicolumn{2}{|l|}{$575(20.4)$} \\
\hline $\mathrm{CC}>50-85$ & \multicolumn{2}{|l|}{$1535(37.0)$} & \multicolumn{2}{|l|}{$1126(39.9)$} \\
\hline $\mathrm{cc}>85$ & \multicolumn{2}{|l|}{$1728(41.7)$} & \multicolumn{2}{|l|}{$1086(38.5)$} \\
\hline extracardiac arteriopathy & \multicolumn{2}{|l|}{$80(1.9)$} & \multicolumn{2}{|l|}{$71(2.5)$} \\
\hline poor mobility & NIM & $94(2.3)$ & NIM & $58(2.1)$ \\
\hline previous cardiac surgery & NIM & $263(6.3)$ & NIM & $75(2.7)$ \\
\hline pulmonary disease & \multicolumn{2}{|l|}{$312(7.5)$} & \multicolumn{2}{|l|}{$218(7.7)$} \\
\hline active endocarditis & NIM & $49(1.2)$ & NIM & $11(0.4)$ \\
\hline critical pre-operative state & NIM & $107(2.6)$ & NIM & $89(3.2)$ \\
\hline diabetes on insulin & NIM & $655(15.8)$ & & $592(21.0)$ \\
\hline neurological disease & $87(2.1)$ & NIM & $56(2.0)$ & NIM \\
\hline reoperation & $1229(29.7)$ & NIM & $811(28.8)$ & NIM \\
\hline $\mathrm{vt} / \mathrm{vf}$ & $15(0.4)$ & NIM & $12(0.4)$ & NIM \\
\hline cpr & $8(0.2)$ & NIM & $6(0.2)$ & NIM \\
\hline iabp & NIM & NIM & & \\
\hline ventilation & $23(0.6)$ & NIM & $16(0.6)$ & NIM \\
\hline nyha i & NIM & $1821(43.9)$ & NIM & $1333(47.3)$ \\
\hline nyha iI & NIM & $1871(45.1)$ & NIM & $1235(43.8)$ \\
\hline
\end{tabular}


M. Azizan Petra et al., Sch J App Med Sci, June, 2020; 8(6): 1390-1396

\begin{tabular}{|c|c|c|c|c|}
\hline nyha iII & NIM & 307 (7.4) & NIM & $156(5.5)$ \\
\hline nyha iV & NIM & $17(0.4)$ & NIM & $8(0.3)$ \\
\hline $\operatorname{ccs} 4$ & NIM & $42(1.0)$ & NIM & $37(1.3)$ \\
\hline unstable angina & $408(9.8)$ & NIM & $369(13.1)$ & NIM \\
\hline lvef $>50 \%$ & \multicolumn{2}{|l|}{$2077(50.1)$} & \multicolumn{2}{|l|}{$1284(45.5)$} \\
\hline lvef $31-50 \%$ & \multicolumn{2}{|l|}{$1531(36.9)$} & \multicolumn{2}{|l|}{$1172(41.6)$} \\
\hline lvef $21-30 \%$ & \multicolumn{2}{|l|}{$274(6.6)$} & \multicolumn{2}{|l|}{$202(7.2)$} \\
\hline lvef $<20 \%$ & \multicolumn{2}{|l|}{$29(0.7)$} & \multicolumn{2}{|l|}{$22(0.8)$} \\
\hline recent mi ( $<90$ days $)$ & \multicolumn{2}{|l|}{$606(14.6)$} & \multicolumn{2}{|l|}{$531(18.8)$} \\
\hline pulmonary hpt (31-55mmh) & NIM & $135(3.3)$ & NIM & $71(2.5)$ \\
\hline pulmonary hpt (>55mmhg) & NIM & $56(1.4)$ & NIM & $10(0.4)$ \\
\hline systolic pressure $>60 \mathrm{mmhg}$ & $23(0.6)$ & NIM & $4(0.1)$ & NIM \\
\hline \multicolumn{5}{|l|}{ urgency } \\
\hline elective & \multicolumn{2}{|l|}{$2911(70.2)$} & \multicolumn{2}{|l|}{$1947(69.0)$} \\
\hline emergency & \multicolumn{2}{|l|}{$112(2.7)$} & \multicolumn{2}{|l|}{$83(2.9)$} \\
\hline urgent & \multicolumn{2}{|l|}{$187(4.5)$} & \multicolumn{2}{|l|}{$170(6.0)$} \\
\hline salvage & \multicolumn{2}{|l|}{-} & \multicolumn{2}{|l|}{-} \\
\hline \multicolumn{5}{|l|}{ planned operation } \\
\hline isolated cabg & \multicolumn{2}{|l|}{$2771(66.9)$} & \multicolumn{2}{|l|}{$2719(96.4)$} \\
\hline single non cabg & \multicolumn{2}{|l|}{$580(14.0)$} & \multicolumn{2}{|l|}{$6(0.2)$} \\
\hline 2 procedures & \multicolumn{2}{|l|}{$585(14.1)$} & \multicolumn{2}{|l|}{$39(1.4)$} \\
\hline 3 procedures & \multicolumn{2}{|l|}{$154(3.7)$} & \multirow{2}{*}{\multicolumn{2}{|c|}{$\begin{array}{l}9(0.3) \\
5(0.5)\end{array}$}} \\
\hline planned surgery on thoracic aorta & \multicolumn{2}{|l|}{$103(2.5)$} & & $15(0.5)$ \\
\hline POSTINFARCT SEPTAL RUPTURE & $4(0.1)$ & NIM & $3(0.1)$ & NIM \\
\hline
\end{tabular}

For the entire cohort (Figure-1), the ROC curve for EuroSCORE was 0.679 (95\% confidence interval (CI): $0.639-0.720] \mathrm{p}<0.001)$; EuroSCORE II was 0.615 (95\% CI: $0.576-0.655) \quad \mathrm{p}<0.001)$. For isolated CABG patients (Figure 2), the ROC curve for EuroSCORE was 0.670 [95\% CI: $0.614-0.726$ ] $\mathrm{p}<0.001$ ); EuroSCORE II was 0.609 (95\% CI: $0.556-$ $0.661), \mathrm{p}<0.001)$.

For the entire cohort, the Hosmer-Lemeshow (HL) goodness-of-fit test showed no significant difference between observed and predicted mortality according to EuroSCORE model (Table-3), (Chi-square $=5.284, \mathrm{P}=0.508$ ) and EuroSCORE II model (Table$4)$, $($ Chi-square $=15.828, \mathrm{P}=0.050)$.
For the subgroup of isolated CABG patients (Table-5), the Hosmer-Lemeshow (HL) goodness-of-fit test showed no significant difference between observed and predicted mortality according to EuroSCORE model $($ Table-5), $($ Chi-square $=5.365, \mathrm{P}=0.498)$ and EuroSCORE II model (Table-6), (Chi-square $=9.839, \mathrm{P}$ $=0.276$ ).

For the entire cohort (Table-7), the observed and predicted mortality were $4.56 \%$ and $3.7 \%$ respectively for EuroSCORE; observed and predicted mortality were similar at $4.56 \%$ for EuroSCORE II. For isolated CABG patients (Table-8), the observed and predicted mortality were $3.62 \%$ and $3.36 \%$ respectively for EuroSCORE; the observed and predicted mortality were $3.62 \%$ and $3.97 \%$ respectively for EuroSCORE II.

Table-3: Goodness-of-fit test for EuroSCORE (Overall)

\begin{tabular}{|l|l|l|l|l|l|}
\hline \multirow{2}{*}{ Risk group } & ALIVE patients & \multicolumn{2}{|l|}{ DEAD patients } & \multirow{2}{*}{ total } \\
\cline { 2 - 5 } & Observed & Expected & Observed & Expected & \\
\hline 1 & 319 & 321.1 & 7 & 4.9 & 326 \\
\hline 2 & 487 & 483.5 & 6 & 9.5 & 493 \\
\hline 3 & 588 & 590.1 & 17 & 14.9 & 605 \\
\hline 4 & 773 & 776.7 & 29 & 25.3 & 802 \\
\hline 5 & 547 & 544.2 & 20 & 22.8 & 567 \\
\hline 6 & 408 & 412.7 & 27 & 22.3 & 435 \\
\hline 7 & 347 & 343.2 & 20 & 23.8 & 367 \\
\hline 8 & 487 & 484.4 & 63 & 65.6 & 550 \\
\hline \multicolumn{7}{|l|}{ p value $=0.508$, Chi-square $=5.284$} \\
\end{tabular}

$* *$ The logistic regression fits the data since H-L test $>0.05$ 
Table-4: Goodness-of-fit test for EuroSCORE II (Overall)

\begin{tabular}{|l|l|l|l|l|l|}
\hline \multirow{2}{*}{ Risk group } & \multicolumn{2}{|l|}{ ALIVE patients } & \multicolumn{2}{l|}{ DEAD patients } & \multirow{2}{*}{ total } \\
\cline { 2 - 5 } & Observed & Expected & Observed & Expected & \\
\hline 1 & 407 & 400.0 & 8 & 15.0 & 415 \\
\hline 2 & 406 & 399.6 & 9 & 15.4 & 415 \\
\hline 3 & 405 & 399.3 & 10 & 15.7 & 415 \\
\hline 4 & 395 & 399.0 & 20 & 16.0 & 415 \\
\hline 5 & 396 & 398.5 & 19 & 16.5 & 415 \\
\hline 6 & 394 & 397.8 & 21 & 17.2 & 415 \\
\hline 7 & 395 & 400.9 & 24 & 18.1 & 419 \\
\hline 8 & 400 & 398.7 & 18 & 19.3 & 418 \\
\hline 9 & 386 & 393.5 & 29 & 21.5 & 415 \\
\hline 10 & 372 & 368.7 & 31 & 34.3 & 403 \\
\hline \multicolumn{7}{|l|}{$p$} & $p$ value $=0.050$, Chi-square $=15.828$ & \\
\hline
\end{tabular}

**The logistic regression fits the data since $\mathrm{H}-\mathrm{L}$ test $>0.05$

Table-5: Goodness-of-fit test for EuroSCORE (Isolated CABG)

\begin{tabular}{|l|l|l|l|l|l|}
\hline \multirow{2}{*}{ Risk group } & ALIVE patients & \multicolumn{2}{|c|}{ DEAD patients } & \multirow{2}{*}{ total } \\
\cline { 2 - 5 } & Observed & Expected & Observed & Expected & \\
\hline 1 & 310 & 311.8 & 6 & 4.2 & 316 \\
\hline 2 & 480 & 476.8 & 5 & 8.2 & 485 \\
\hline 3 & 385 & 387.5 & 11 & 8.5 & 396 \\
\hline 4 & 437 & 441.7 & 17 & 12.3 & 454 \\
\hline 5 & 341 & 338.9 & 10 & 12.1 & 351 \\
\hline 6 & 254 & 253.5 & 11 & 11.5 & 265 \\
\hline 7 & 210 & 209.8 & 12 & 12.2 & 222 \\
\hline 8 & 301 & 297.9 & 30 & 33.1 & 331 \\
\hline
\end{tabular}

$\mathrm{p}$ value $=0.498$ Chi-square $=5.365$

**The logistic regression fits the data since H-L test $>0.05$

Table-6: Goodness-of-fit test for EuroSCORE II (Isolated CABG)

\begin{tabular}{|l|l|l|l|l|l|}
\hline \multirow{2}{*}{ Risk group } & ALIVE patients & \multicolumn{2}{|l|}{ DEAD patients } & \multirow{2}{*}{ total } \\
\cline { 2 - 5 } & Observed & Expected & Observed & Expected & \\
\hline 1 & 293 & 290.4 & 6 & 8.6 & 299 \\
\hline 2 & 278 & 273.6 & 4 & 8.4 & 282 \\
\hline 3 & 277 & 273.5 & 5 & 8.5 & 282 \\
\hline 4 & 273 & 273.3 & 9 & 8.7 & 282 \\
\hline 5 & 271 & 273.1 & 11 & 8.9 & 282 \\
\hline 6 & 270 & 273.7 & 13 & 9.3 & 283 \\
\hline 7 & 268 & 272.3 & 14 & 9.8 & 282 \\
\hline 8 & 269 & 271.6 & 13 & 10.4 & 282 \\
\hline 9 & 273 & 270.5 & 9 & 11.5 & 282 \\
\hline 10 & 246 & 246.0 & 18 & 18.0 & 264 \\
\hline \multicolumn{7}{|l|}{ p value $=0.276$ Chi-square $=9.839$} & \\
\hline
\end{tabular}

**The logistic regression fits the data since H-L test $>0.05$

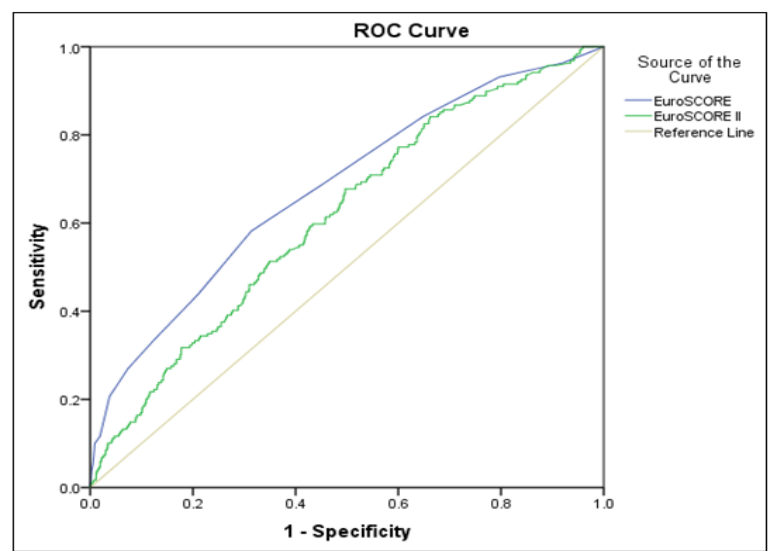

Fig-1: ROC curves for EuroSCORE and EuroSCORE II (Overall) 
M. Azizan Petra et al., Sch J App Med Sci, June, 2020; 8(6): 1390-1396

\begin{tabular}{|l|l|l|l|l|l|}
\hline \multicolumn{5}{|l|}{ Area Under the Curve } \\
\hline Test Result Variable(s) & Area & $\begin{array}{l}\text { Std. } \\
\text { Error }\end{array}$ & $\begin{array}{l}\text { Asymptotic } \\
\text { Sig. }\end{array}$ & $\begin{array}{l}\text { Asymptotic } \\
\text { Interval }\end{array}$ & 95\% Confidence \\
\cline { 3 - 6 } & & .021 & .000 & .639 & .720 \\
\hline EuroSCORE & .679 & .020 & .000 & .576 & .655 \\
\hline EuroSCORE II & .615 & Lower Bound & Upper Bound \\
\hline a. Under the nonparametric assumption \\
\hline b. Null hypothesis: true area =0.5
\end{tabular}

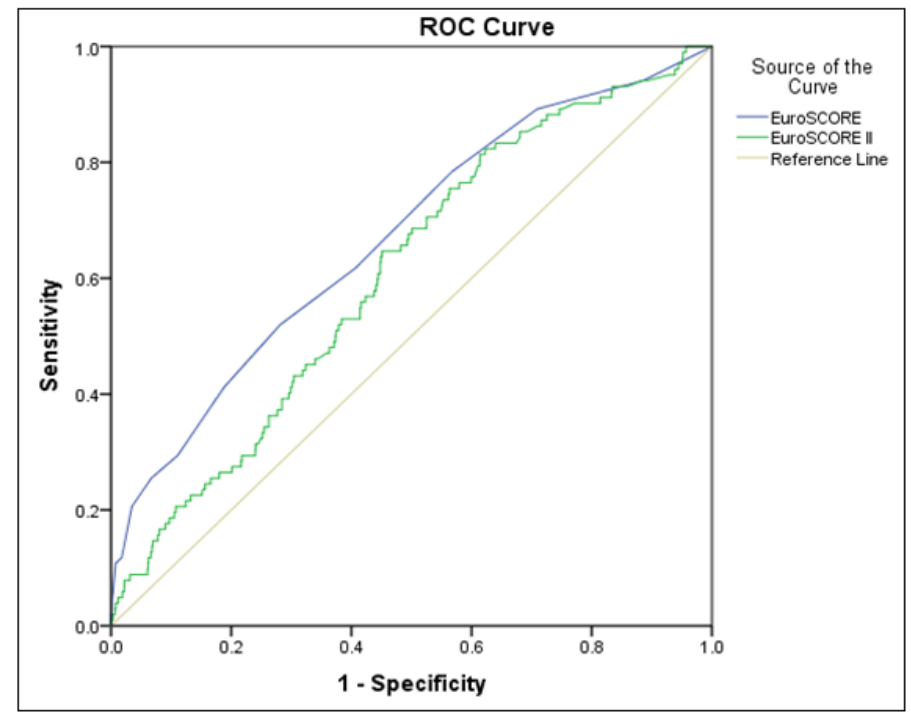

Fig-2: ROC curves for EuroSCORE and EuroSCORE II (Isolated CABG)

\begin{tabular}{|c|c|c|c|c|c|}
\hline \multicolumn{6}{|c|}{ Area Under the Curve } \\
\hline \multirow[t]{2}{*}{ Test Result Variable(s) } & \multirow[t]{2}{*}{ Area } & \multirow[t]{2}{*}{ Std. Error ${ }^{a}$} & \multirow[t]{2}{*}{ Asymptotic Sig. } & \multicolumn{2}{|c|}{ Asymptotic 95\% Confidence Interval } \\
\hline & & & & Lower Bound & Upper Bound \\
\hline EuroSCORE & .670 & .028 & .000 & .614 & .726 \\
\hline EuroSCORE II & .609 & .027 & .000 & .556 & .661 \\
\hline \multicolumn{6}{|c|}{ a. Under the nonparametric assumption } \\
\hline b. Null hypothesis: true & $\mathrm{ea}=0$. & & & & \\
\hline
\end{tabular}

Table-7: Observed and predicted mortality by EuroSCORE and EuroSCORE II risk level for all cohort

\begin{tabular}{|l|l|l|l|}
\hline Risk group & Patients (death) & Observe death (\%) & Predicted death (\%) \\
\hline Low risk (0-2) & $1424(30)$ & $2.11(1.36-2.86)$ & $1.20(1.16-1.24)$ \\
\hline Medium risk (3-5) & $1804(76)$ & $4.21(3.28-5.14)$ & $3.80(3.76-3.83)$ \\
\hline High risk (>=6) & $917(83)$ & $9.05(7.19-10.91)$ & $7.42(7.31-7.53)$ \\
\hline Total & $4145(189)$ & $4.56(3.92-5.20)$ & $3.70(3.63-3.78)$ \\
\hline
\end{tabular}

EuroSCORE

\begin{tabular}{|l|l|l|l|}
\hline Risk group & Patients (death) & Observe death (\%) & Predicted death (\%) \\
\hline Low risk $(<=1.22)$ & $412(8)$ & $1.94(0.61-3.27)$ & $0.89(0.87-0.92)$ \\
\hline Medium risk (1.23-2.02) & $988(25)$ & $2.53(1.55-3.51)$ & $1.62(1.60-1.63)$ \\
\hline High risk $(2.03-4.11)$ & $1229(59)$ & $4.80(3.60-6.00)$ & $2.93(2.90-3.00)$ \\
\hline Very high risk (4.12-47.6) & $1516(97)$ & $6.40(5.17-7.63)$ & $8.78(8.48-9.07)$ \\
\hline Total & $4145(189)$ & $4.56(3.92-5.20)$ & $4.56(4.41-4.70)$ \\
\hline
\end{tabular}

EuroSCOREII

Table-8: Observed and predicted mortality by EuroSCORE and EuroSCORE II risk level for isolated CABG

\begin{tabular}{|l|l|l|l|}
\hline Risk group & Patients (death) & Observe death $(\%)$ & Predicted death $(\%)$ \\
\hline Low risk (0-2) & $1197(22)$ & $1.84(1.08-2.60)$ & $1.07(1.02-1.11)$ \\
\hline Medium risk (3-5) & $1070(38)$ & $3.55(2.44-4.66)$ & $3.82(3.78-3.87)$ \\
\hline High risk (>=6) & $553(42)$ & $7.59(5.38-9.80)$ & $7.42(7.27-7.56)$ \\
\hline Total & $2820(102)$ & $3.62(2.93-4.31)$ & $3.36(3.26-3.45)$ \\
\hline
\end{tabular}

EuroSCORE 


\begin{tabular}{|l|l|l|l|}
\hline Risk group & Patients & Observe death $(\%)$ & Predicted death $(\%)$ \\
\hline Low risk $(<=1.22)$ & $305(6)$ & $1.97(0.41-3.53)$ & $0.90(0.87-0.92)$ \\
\hline Medium risk (1.23-2.02) & $757(13)$ & $1.72(0.79-2.65)$ & $1.62(1.61-1.64)$ \\
\hline High risk (2.03-4.11) & $863(39)$ & $4.52(3.13-5.91)$ & $2.93(2.89-2.97)$ \\
\hline Very high risk (4.12-47.6) & $895(44)$ & $4.92(3.50-6.34)$ & $8.00(7.66-8.34)$ \\
\hline Total & $2820(102)$ & $3.62(2.93-4.31)$ & $3.97(3.82-4.12)$ \\
\hline
\end{tabular}

EuroSCOREII

\section{DISCUSSION}

Risk stratification and risk scoring systems in adult cardiac surgery are becoming important as they provide reliable estimations of the risks associated with surgical procedure and they permit, in some cases to a certain extent, comparison of outcomes among institutions and surgeons by adjusting for a variable mixture of cases. In addition, these models may also provide a more accurate assessment of the indication for surgery in each individual patient which in turn can facilitate a more precise balance between the potential risks and benefits. This is an important role of risk prediction models in cardiac surgery, as not only it will help us to decide surgery suitability, it will also help family and patients to have improved informed consent [2].

However, risk prediction models may not be suitable to certain groups of our patient population. Ready-made models may not accurately predict local outcomes and will require validation prior to use [1]

We have shown that in this study that both models do not predict outcomes accurately in the entire cohort. Even though both risk prediction models represented under-prediction rates for the entire cohort, Euroscore II appears to perform better at risk prediction with an under-prediction rate of only $2 \%$ when compared to Euroscore which produced an underprediction rate of $20 \%$.

However, it is important to note that the calibration which is calculated by the HosmerLemeshow (HL) goodness-of-fit test show good results confirming statistical precision with $\mathrm{P}$ values of $>0.05$. However, despite still showing discriminatory ability, this study shows poor discriminatory abilities for both risk models where the ROC curve values were 0.609 0.679 .

For both models, the under-prediction rates may be due to several factors. The first factor could be due to the difference in the underlying co-morbidities between Asian and European populations. For example, higher prevalence in diabetes and hypertension [10]. The other factor could be information bias; some information were not disclosed by patients for example family history and smoking.

The major limitation of this study is that it is done only as a single centre study. Although this study was done from a high-volume institution, it is still important to consider the practice of other institutions. This data has not been validated externally. Other than that, this study could have been done over a longer time period. The other limitation of the study is its retrospective nature which may introduce potential bias; particularly in regards to the accuracy of data which relied on individuals recalling the information.

\section{CONCLUSION}

Euroscore and Euroscore II is relatively a simple tool to be used for risk stratification. It is an easy scoring system including most of the usual risk factors [6]. However, despite poor discrimination under the ROC curve, the calibration of both models was good and acceptable to be used for risk prediction tools in our centre. However, we still should be cautious in the utilization of these risk stratification models. The differences in demographic characteristics should be respected among different population groups.

\section{REFERENCES}

1. Yap CH, Reid C, Yii M, Rowland MA, Mohajeri M, Skillington PD, Seevanayagam S, Smith JA. Validation of the EuroSCORE model in Australia. European journal of cardio-thoracic surgery. 2006 Apr 1;29(4):441-6.

2. Barili F, Pacini D, Capo A, Rasovic O, Grossi C, Alamanni F, Di Bartolomeo R, Parolari A. Does EuroSCORE II perform better than its original versions? A multicentre validation study. European heart journal. 2013 Jan 1;34(1):22-9.

3. Nashef SA, Roques F, Michel P, Gauducheau E, Lemeshow S, Salamon R, EuroSCORE Study Group. European system for cardiac operative risk evaluation (Euro SCORE). European journal of cardio-thoracic surgery. 1999 Jul 1;16(1):9-13.

4. Michel P, Roques F, Nashef SA, EuroSCORE Project Group. Logistic or additive EuroSCORE for high-risk patients?. European Journal of Cardio-thoracic surgery. 2003 May 1;23(5):684-7.

5. Nashef SA, Roques F, Hammill BG, Peterson ED, Michel P, Grover FL, Wyse RK, Ferguson TB. Validation of European system for cardiac operative risk evaluation (EuroSCORE) in North American cardiac surgery. European journal of cardio-thoracic surgery. 2002 Jul 1;22(1):101-5.

6. Sergeant, P., de Worm, E., \& Meyns, B. (2001). Single centre, single domain validation of the EuroSCORE on a consecutive sample of primary 
and repeat CABG. European journal of cardiothoracic surgery, 20(6), 1176-1182.

7. Garcia-Valentin A, Mestres CA, Bernabeu E, Bahamonde JA, Martín I, Rueda C, Domenech A, Valencia J, Fletcher D, Machado F, Amores J. Validation and quality measurements for EuroSCORE and EuroSCORE II in the Spanish cardiac surgical population: a prospective, multicentre study. European Journal of CardioThoracic Surgery. 2016 Feb 1;49(2):399-405.

8. Amr MA, El-shorbagy AA. Evaluation of accuracy of EuroSCORE II in prediction of inhospital mortality in patients underwent mitral valve replacement in Egypt. Journal of the Egyptian Society of Cardio-Thoracic Surgery. 2016 Aug 1;24(2):135-42.

9. Akar AR, Kurtcephe M, Sener E, Alhan C, Durdu S, Kunt AG, Güvenir HA. Validation of the EuroSCORE risk models in Turkish adult cardiac surgical population. European journal of cardiothoracic surgery. 2011 Sep 1;40(3):730-5.

10. Zheng Z, Li Y, Zhang S, Hu S. The Chinese coronary artery bypass grafting registry study: how well does the EuroSCORE predict operative risk for Chinese population?. European journal of cardio-thoracic surgery. 2009 Jan 1;35(1):54-8. 